\title{
August Wilhelm Schlegels Rezeption spanischer Literatur
}

Strosetzki, Christoph

First published in:

Der Europäer August Wilhelm Schlegel, S. 143 - 157, de Gruyter, Berlin 2010, ISBN 978-3-11-022846-5

Münstersches Informations- und Archivsystem multimedialer Inhalte (MIAMI) URN: urn:nbn:de:hbz:6-92419438812 
Christoph Strosetzki (Münster)

\section{August Wilhelm Schlegels Rezeption spanischer Literatur}

August Wilhelm Schlegels Einschätzung der spanischen Literatur ist eng mit seinem Spanienbild verbunden, dessen zeitgenössische Ausprägung den Ausführungen zu Schlegels eigener Konzeption kurz vorangestellt werden soll. Im Anschluss daran sollen die zentralen literarischen Gattungen, Werke und Tendenzen vorgeführt werden, die Schlegel in der spanischen Literatur bewundert. Spanien ist für ihn das Land, wo eine „brennende Leidenschaftlichkeit" jeden Gegenstand zum sinnlichen Genuss macht und adelt. ${ }^{1}$ Allgemein stellt Schlegel fest, dass die nördlichen Nationen nicht viel von den südlichen Völkern Europas hören, weil dort die Druckerpresse und der Buchhandel weniger aktiv seien. Dieser scheinbare Stillstand oder diese Lethargie sei aber eher ein Vorteil, so wie es ein Vorteil sein kann, die schlechte Witterung verschlafen zu haben. Da Schlegel damit die. Aufklärung des 18. Jahrhunderts meint, ist es verständlich, dass er es ablehnt, dass die Spanier sich an der Autorität der Engländer und Franzosen orientieren. ${ }^{2}$

Schlegel schrieb seinen Aufsatz Über das spaniscbe Theater im Jahr 1803, ein Thema, dem er sich bei Mme de Staël, der er 1804 nach Coppet folgte, mangels Zeit und Büchern weniger widmete. ${ }^{3}$ Im Rahmen seiner sich anschließenden Tätigkeit an der Universität Bonn von 1818 bis 1845 war er „der Repräsentationsprofessor, der bei allen feierlichen Gelegenheiten als Redner und Dichter in lateinischer und deutscher Sprache herausgestellt wurde “.4 Sein Bonner Kollege Friedrich Diez.(1784-1876), bekannt als Gründer der Romanischen Philologie, erhielt in Bonn ab 1830 eine

1 August Wilhelm Schlegel, „Vorlesungen über dramatische Kunst und Literatur. 2. Teil“", in Lohner VI, S. 264

August Wilhelm Schlegel, „Vorlesungen über Ästhetik [1803-1827]“, in: KAV II/1, hier S. 211 .

3 Vol. Hilde Marianne Paulini, August Wilhelm Schlegel und die Vergleichende Literaturyissenschaft Frankfurt/Main 1985, S. 255

4 Walter R. Schirmer, ,August Wilhelm von Schlegel. 1767-1845“, in: Bonner Gelebrte. Beitragge zut Geschichte der Wissensshaffen in Bonn. Sprachwissenschaffen, Universität Bonn (Htsg.), Bonn 1970, S. 11-20, hier S. 14 
Professur, nachdem er dort seit 1821 als Lektor Spanisch, Italienisch und Portugiesisch lehrte. Dass er nicht auch das Französische vertrat, lässt sich auf die antifranzösische Haltung seiner Zeit zurückführen, die sich gegen Napoleon wandte. Bereits 1808 greift Johann Gottlieb Fichte in seinen Reden an die deutscbe Nation die „Neulateinet" an und meint damit die Franzosen, deren Wesen steril, oberflächlich, unernst und faul sei, während der germanische Chatakter tief, gemütvoll, emst und fleißig sei. Nur das Mittelalter sei in der Romania von Interesse, da es germanisch geprägt sei, wie die Ritterepik zeige, in Spanien aber noch stärker als in Frankreich. So ist es nicht erstaunlich, dass auch Diez in seiner Besprechung von Jacob Grimms Silva de romances viejos 1815 etfreut in den Romanzen den Sagenkreis über Karl den Großen und seine zwölf Genossen, den Geist der gotisch-germanischen Feudalordnung und tiefes deutsches Gemüt entdeckt, während er im spanischen Heldenepos Cid gat den Nibelungengeist verspürt. Nicht selten ist es in der Geschichte der Romanistik die Abwendung von Frankreich, die eine Hinwendung zu Spanien mit sich bringt. Bereits 1806/07 hatte man in der Auseinandersetzung mit dem französischen Klassizismus, det französischen Aufklärung und mit der Französischen Revolution jene für Deutschland charakteristischen Stereotypen, wie Einfühlung, Naturgefühl, Drang ins Unendliche, Treue zum Alten, Arbeitsamkeit, Sachlichkeit, Heiligung der Weiblichkeit, Freiheitsliebe und Schicksalsglaube entwickelt, die noch 1927 Eduard Wessler in Esprit und Geist. Versuch einer Wesenskunde des Deutscben und des Franzosen den entsprechenden französischen entgegensetzte. ${ }^{5}$ Es fällt auf, wie sehr einige dieser "deutschen" Stereotypen jenen ähneln, die die deutsche Romantik den Spaniern zuschrieb.

Die allgemeine Spanienbegeisterung in der zweiten Hälfte des 18. Jahrhunderts bedeutete einen Impuls auch für außeruniversitäre hispanistische Studien. Ansätze des im deutschen Sprachraum aufkeimenden Spanieninteresses belegen allerdings schon frühere Übersetzungen, Anthologien und Studien. ${ }^{6}$ Als Beispiele seien genannt: Gotthold Ephraim

5 Vgl. Michael Nerlich, „Romanistik: Von dex wissenschaftlichen Kriegsmaschine gegen Frankreich zur komparatistischen Konsolidierung der Frankreichforschung “, in: Romanisht ci. S. 404 40, vgl. auch Frank-

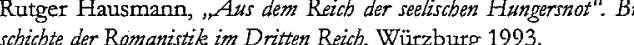

Vgl Gerart Hoffmeister, Spmim um Douts 1993.

Vgl. Gethart Hoffmeister, Spanien znd Deutscbiand. Geschicbte und Doktmentation der literarischen Bezziebungen, Berlin 1976, S. 86ff.; Manfred Tietz (Hrsg.), Das Spanieninteresse im deutschen
Spracbraum. Beitrage zur Geschicbte der Hippanistik vor 1900, Frankfurt/Main 1989; Ludwig Spracbraum. Beiträge zur Geschicbte der Hispanistik vor 1900, Frankfurt/Main 1989; Ludwig
Schrader, „El interés por el mundo ibético y los otígenes del hispanismo cient́fico en los paises de lengua alemana (siglo XIX)", in: Christoph Strosetzki/Jean-Francois Botrel/Manfred Tietz (Hrsg.), Actas del I Enctentro Franco-Alemán de Hippanistas (Mainz 9-123.89) Frankfurt/Main 1991, S. 1-18.
Lessings Hamburgische Dramaturgie (1767/69), ${ }^{7}$ in der ex die Mischung von Tragischem und Komischem durch den gracioso bei Lope de Vega als nachahmenswerte Alternative zum klassisch-französischen Theater sicht, seine 1752 veröffentlichte Übersetzung von Juan Huartes Examen de ingenios para las ciencias, Johann Andreas Diezes 1769 veröffentlichte Bearbeitung der spanischen Literaturgeschichte des Luis José Velázquez, Friedrich Justin Bertuchs 1775/77 herausgegebene Don Quijote-Übersetzung und sein von 1780 bis 1782 herausgegebenes Magazin der spaniscben und portugiesischen Literatur. Johann Gottfried Herder beschäftigte sich mit den spanischen Romanzen, da in ihnen gemäß seiner Vorstellung vom „Volkslied“ die Eigenart der Volksseele zum Ausdruck komme. So veröffentlichte er in seiner Sammlung der Volkslieder (1778/79) neben Romanzen aus dem Mittelalter auch fünf von Góngora verfasste Romanzen. Auch im Don Quijote sah Herder die spezifische Denkweise des spanischen Volkes ausgedrückt. Ludwig Tieck übersetzte von 1799 bis 1801 Don Quijote und machte August Wilhelm Schlegel mit Calderón bekannt. Goethe hatte Cervantes gelesen und geschätzt. 1802 überreichte ihm August Wilhelm Schlegel seine Übersetzung von Calderóns Andacht zum Krewre, ein Jahr später den ersten Band seiner Sammlung Spanisches Theater. ${ }^{8}$ Nach seiner Anstellung als Theaterdirektor in Weimar im Jahr 1791 lässt Goethe von Calderón 1811 den Standbaften Prinzen, 1812 Das Leben ein Traum und 1815 die Große Zenobia aufführen. ${ }^{9}$ Ihn fasziniert das Fremde, Mittelalterliche, Arabische bei Calderón: „Nur wer Hafis liebt und kennt / Weiß was Calderon gesungen ". ${ }^{10}$ Friedrich Schiller sieht in Cervantes' Don Quijote und im Gegensatz zwischen dem edlen Idealisten Don Quijote und Sancho als dem Vertreter der prosaischen Wirklichkeit ein Paradebeispiel für die sentimentalische Dichtung: „Der sentimentalische Dichter hat es daher immer mit zwei streitenden Vorstellungen und Empfindungen, mit der Wirklichkeit als Grenze und mit seiner Idee als dem Unendlichen zu tun"."11 Die Aufrechterhaltung der Spannung zwischen Wirklichkeit und

7 Zur Wiederentdeckung der spanischen Literatur bei Lessing: Vgl. Hoffmeister, Spanien und Deutschland, (Anm. 6), S. 89-91.

Zin Schlegels Konzeption von Übersetzung vgl.: Paulini, Literatunvissenschaff, (Anm. 3), S. 151-158.

9 Vgl. Hoffmeister, Spanien und Deutscbland, (Anm. 6), S. 109.

10 Johann Wolfgang Goethe, "West-östlicher Divan. Teil I", in: Goethe FA MI/1, hier S. 66 ; vgl. auch: Brief an Gries vom 29.5.1816; zur franösischen, orientalisch orept, ho lung eines ,andalusischen Paradieses" vgl. Friedrich Wolfzettel, Spanien als europösischer Orient und die (romantische) Andalusienreise: Edgat Ouinets Mes vacances en Kontext", in: Werner Helmich/Helmut Meter/Astrid Poier-Bernhard (Hrsg) Espagne im Umbrïbbe. Romanistische Studien zu Ehren won Ulrich Scbulz-Buscbhatss, München 2002, S. 90104.

11 Friedrich Schiller, Ausgewzäbite Werke, Bd. 5, Ernst Müller (Hrsg.), Darmstadt 1954, S. 409. 
Ideal sei charakteristisch für moderne satirische Dichtung. Schopenhauer übersetzt 1832 Baltasar Graciáns Oráculo manual. Es zeigt sich also bei den Autoren der deutschen Klassik und Romantik eine deutliche Bevorzugung des Mittelalters und des Siglo de Oro (16. und 17. Jahrhundert).

Doch zurück zu August Wilhelm Schlegel. Seine Vorliebe gilt dem spanischen Mittelalter und dem mittelalterlichen Rittertum: „Nirgends hatte der ritterliche Geist die politische Existenz des Rittertums länger überlebt als in Spanien". ${ }^{12}$ Der Kampfgeist des Rittertums prägt folglich auch die Dichtung. Anders als im übrigen Europa waren die Dichter nicht Höflinge, Gelehrte oder Bürger, sondern kämpfende Ritter und verbanden „armas y letras“".13 Der Ritter hatte einen Sinn für Gerechtigkeit und griff Schwächere nicht an. ${ }^{14} \mathrm{Da}$ sich die spanischen Ritter nach Schlegel vom achten bis zum fünfzehnten Jahrhundert gleichsam in einem großen Kreuzzug befanden, sollte der Krieg gleichzeitig das verloren gegangene Staatsgebiet wiedererlangen und der Verbreitung des christlichen Glaubens dienen. ${ }^{15}$ Wichtiges Element im Tugendsystem der Ritter war daher die Frömmigkeit. ${ }^{16}$ Hinzu kam zu den Pflichten und Ehren die Ehrerbietung gegenüber der Geistlichkeit, Respekt gegenüber Höhergestellten und Untergebenen, besonders aber Höllichkeit gegenüber der Frau. An anderer Stelle nehmen die Spitzenpositionen bei den Tugenden die Tapferkeit und Tüchtigkeit des Mannes, verbunden mit Treue und Redlichkeit, ein.

Die positiven Eigenschaften, die Schlegel den spanischen Rittern zuschreibt, sind eben diejenigen, die er auch bei seinen eigenen deutschen Vorfahren lobt. Dazu gehört das Gefühl für Gleichheit, die Tapferkeit im Krieg, die Lust an Kämpfen, seien dies Zweikämpfe zur Erlangung von Gottesurteilen oder Jagd und Waffenübungen in der Friedenszeit. ${ }^{17}$ Schon bei den römischen Historikern galten die Germanen als Stamm von Riesen, ein Faktum, das nach Schlegel seine Bestätigung auch in der Größe der germanischen Rüstungen findet. $\mathrm{Da}$ die Goten einen germanischen Stamm bilden, der in der spanischen Geschichte eine prägende Rolle hatte, lässt sich die Frühgeschichte der Spanier als deutsche Geschichte interpretieren. So könne man in der würdevollen kastilischen Sprache noch "die tauhe Kraft und die Treuherzigkeit der Goten" erkennen. ${ }^{18}$ Zwei Grundprinzipien Europas unterscheidet Schlegel: erstens das Christentum,

12 Lohner VI, (Anm. 1), S. 262

13 Ebd.

$14 \mathrm{KAV} \mathrm{II/1,} \mathrm{(Anm.} \mathrm{2),} \mathrm{S.} 81$.

15 August Wilhelm Schlegel, „Vorlesungen über Encyklopädie [1803]“, in: KAV III, hier S. 231.

$16 \mathrm{KAV}$ II/1, (Anm. 2), S. 80

17 Ebd., S. $75 f$

18 Lohnet VI, (Anm. 1), S. 261 „dās andere Element war die Deutsche Stammesart. Aus diesem beydem zusammen mit den Trümmern des classischen Altertums muß die neuere Geschichte construiert werden". 19 Wann beginnt die "neuere Geschichte"? Auch hier bleibt Schlegel keine Antwort schuldig: „Die Völkerwanderung ist folglich, materiell genommen, die Epoche der neueren Geschich-

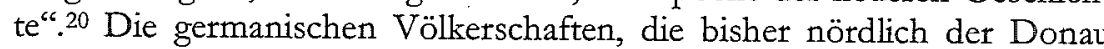
und östlich des Rheins gewohnt hatten, teilten sich nach Schlegel in zwei Richtungen: „die eine naç Süden, nämlich nach Italien, Gallien und Spanien; die andere nach Norden, wo ich zuerst Britannien rechne"“.21

Die Gotenherrschaft in Spanien, das sieht Schlegel durchaus richtig, endet im achten Jahrhundert durch die Eroberungen der Araber, die das westgotische Königreich stürzten und ganz Spanien eroberten mit Ausnahme einiger Gebirgsgegenden im Norden, „und von wo aus bald durch Zurückdrängen der Araber die Königreiche Castilien und Aragon gestiftet wurden“.22 Für Spanien kämpfen hieß nun für das Christentum kämpfen. ${ }^{23}$ Den Gegensatz zwischen Morgenland und Abendland charakterisiett Schlegel als unversöhnlich und in Politik, Mythologie und Literatur präsent:

Die Entgegensetzung der Religionen war freylich in det Erscheinung oben auf, mit derselben verband sich aber eine politische: in Europa waren die Maximen republicanisch unter der Form der Feudalität, im Orient despotisch. [...] Europa focht gegen die Saracenen, in denen es mit Wahrheit seine Erbfeinde erkannte, nicht nur für seine religiöse, sondern auch für seine politische Existenz. [...] Dieser Gegensatz zwischen Christlich und Saracenisch spielte in der ganzen RitterMythologie eine große Rolle, er ist einer von den Cardinalpunkten, um die sich viele Begebenheiten drehen. [...] In den Spanischen Dichtern findet man den Contrast zwischen dem südlich Occidentalischen und dem Orientalischen [...] sowohl in historisch erfundenen Darstellungen wunderwürdig benutzt, in vielen Novellen, Romanzen und Dramen, und diese nahe Gegenwart des beständigen Antagonisten ethöht das nationale Bewusstsein. ${ }^{24}$

Die unversöhnliche Gegensätzlichkeit zeigt sich auch dort, wo Schlegel die Rolle der Spanier als Vorwacht des bedrohten Europa gegen die Einbrüche der Araber betont. Er hält es daher auch für unmöglich,

dass die feinere Bildung ritterlicher Sitten zuerst von den Arabern auf die Europäer übergegangen sey. Vielmehr mag der lange Aufenthalt jener im Occident, und ihr langer, wenn auch meistens kriegerischer Verkehr mit diesen

$19 \mathrm{KAV}$ II/1, (Anm. 2), S. 67.

20 Ebd., S. 67.

21 Ebd.

$22 \mathrm{KAV} \mathrm{\amalg I,} \mathrm{(Anm.} \mathrm{15),} \mathrm{S.} 229$.

23 Vgl. Silke Agnes Reavis, August Wilhelm Scblegels Auffassung der Tragödie im Zusammenbang mit seiner Poetike und ärtbetischen Theorien seiner Zeit, Frankfurt/Main 1978, S. 134

$24 \mathrm{KAV} \Pi / 1$, (Anm. 2), S. 70f 
bey den Arabern etwas von ritterlichen Tugenden geweckt, vielleicht auch die orientalische Sclaverey des anderen Geschlechts dort gemildert haben. ${ }^{25}$

Systematisch allerdings erscheint Schlegel der Islam dem Christentum vergleichbar,

als beyde gegen Heidenthum, d. h. nationale Mytholgie und symbolische Naturreligion gerichtet waren. Innerhalb dieser Gleichartigkeit bildeten sie aber wieder den vollkommensten Gegensatz: das Christenthum ist der idealistische, der Mohamedanismus der realistische Monotheismus. [...] Von jeher war nämlich im Orient der Idealismus, im Occident der Realismus einheimisch. ${ }^{26}$

Damit nimmt Schlegel erneut eine aus seiner Perspektive negative Bewertung des Islam vor.

Nach der Vertreibung der Araber kann Schlegel eine Blütezeit Spaniens im 17. Jahrhundert konstatieren: „Die verschiedenen Bildungsepochen der spanischen Blütezeit lassen sich mit den Namen dreier berühmter Schtiftsteller, des Cervantes, Lope de Vega und Caiderón bezeichnen'. ${ }^{27}$ Gerade mit letzterem hat nach Schlegel das romantische Schauspiel der Spanier den Gipfel der Vollendung erreicht. ${ }^{28}$ Da durch das politische Übergewicht Spaniens im sechzehnten Jahrhundert die Kenntnis der spanischen Sprache sehr verbreitet worden war, kann Schlegel noch in der ersten Hälfte des 17. Jahrhunderts viele Spuren von Vertrautheit mit der spanischen Literatur in Frankreich, Italien, England und Deutschland konstatieren. Erst am Ende des 17. Jahrhunderts sieht Schlegel Spanien am Ende: „Das Gefühl der National-Ehre ging verlohren, die Regierung behandeite die Unterthanen nach den französischen Grunđsätzen [...], der französische Einfluss war seitdem vorwaltend".29 Wobei, er an anderer Stelle die Meinung vertritt, Spanien habe die Aufklärung verschlafen und diese sei „bloß eine nordische Eigenheit“.30 Jedenfalls mahnt Schlegel bei der Einschätzung der geschichtlichen Leistung der Spanier zur Vorsicht. Spanien sei keine „schwächere Kopie der tonangebenden Europäischen Staaten. [...] Die Geschichte Spaniens ist durch die protestantischen Historiker unglaublich entstellt und eigentlich keine Nation so schimpflich verläumdet worden". 31

Poesie ist nach Schlegel etwas typisch Spanisches. Gerade bei den Spaniern könne die Poesie nicht aussterben, da die Spanier noch immer die Stücke ihrer alten großen Meister lesen. Klar ist aber für Schlegel, dass

25 KAV III, (Anm. 15), S. 231f

26 KAV II/1, (Anm. 2), S. 69.

27 KAVII, (Anm. 2), S. 69.

28 Vgl. Reavis, Scblegels Auffassing

ing der Träidie (Anm. 23) S. 133

29 KAV III (Anm 15), S. 230

Ebd., S. 232.

31 Ebd., S. $230 \mathrm{f}$. die Poesie sich in ihren materiellen Manifestationen im Laufe der Geschichte verändert und ,aus den Nahrungsstoffen eines veränderten Zeitalters sich auch einen anders gestalteten Leib zubilden muss".32 Schlegel unterstreicht ihre Bedeutung und ihre Beziehung zu verwandten Wissenschaften: Sie sei

den vier Elementen zu vergleichen. Die Religion ist das Feuer [...] Die Sittlichkeit das Wasser [...] Die Wissenschaft die Erde [...]. Die Poesie endlich ist der Luft zu vergleichen, dem Anschein nach ein bloß spielendes und ergötzliches Element, das in gelinden Zephyrn Blumendüfte, die geistigen Ausflüsse zarter Körper, herbeiführt, aber im unbewußten Athmen zum Leben unentbehrlich ist. ${ }^{33}$

Das heißt zusammengefasst: „Aechte Poesie wird von selbst zugleich philosophisch, moralisch und religiös sein". 34

Die Konzeption des Romantischen ist verwandt mit der der Poesie. Dante, Petrarca und Boccaccio sind für Schlegel die drei "Stifter" der tomantischen Kunst, bei der die Gattung des Romans dominiere, so dass auch die spanischen Komödien nicht nur romantisch, sondern auch romanhaft seien. ${ }^{35}$ Er beschreibt das romantische Drama als ein großes Gemälde,

wo außer der Gestalt und Bewegung in reicheren Gruppen auch noch die Umgebung der Personen mitabgebildet ist, nicht bloß die nächste, sondern ein bedeutender Ausblick in die Ferne, und dies alles unter einer magischen Beleuchtung welche den Eindruck so oder anders bestimmen hilft. Ein solches Gemälde wird weniger vollkommen begrenzt sein als die Gruppe, denn es ist ein ausgeschnitteneniger vollkommen begrenzt sein als die Gruppe, denn es

Das einzelne hat also im Ganzen zu stehen. Schlegel hebt die Rechte der Poesie und die Natur des romantischen Dramas hervor,

welches eben, weil es pittoresk ist und sein soll, reichere Umgebungen und Kontraposte für seine Hauptgruppen erfordert. In aller Kunst und Poesie, vornehmlich aber in der romantischen, macht die Phantasie als eine unabhängige Seelenkraft, die sich nach eigenen Gesetzen regiert, ihre Ansprüche geltend. ${ }^{37}$

$\mathrm{Zu}$ den Gegensätzen von Nähe und Ferne, die die romantische Kunst mischt, kommen weitere unauflösliche Mischungen:

32 Lohner VI, (Anm. 1), S. 110

33 KAVTH/1, (Anm 2$),$ S. 110.

34 Ebd., S. 217.

35 Annelen Grosse-Brockhoff, Das Konzept des Klassischen bei Friedricb und August Willbelm Scblegel, Köln 1981, S. 223-224.

36 Lohner VI, (Anm. 1), S. 112

37 Vgl. ebd. S. 128; Zum Pittoresken vgl. Grosse-Brockhoff, Das Konzept des Klassischen, (Anm 35) S. 228-230. 
[A]lle Entgegengesetzten, Natur und Kunst, Poesie und Prosa, Ernst und Scherz, Erinnerung und Ahnung, Geistigkeit und Sinnlichkeit, das Irdische und Göttliche, Leben und Tod, verschmilzt sie auf das innigste miteinander. ${ }^{38}$

Hinzu kommen im romantischen Drama nach Schlegel die Mischung von Scherz und Ennst und die Mischung der dialogischen und lyrischen Bestandteile. ${ }^{39}$

Eine typische Gattung der Literatur Spaniens ist nach Schlegel die Romanze. Er betont $z$ war, dass es Volkstomanzen auch bei den Engländern, den Schotten, den Dänen und den Deutschen gebe, bei den Spaniern aber seien sie von verschiedener und eigener Gestaltung. Auch der Name Romanze ,dürfte auf die Gattung allerdings von den Spaniern zuerst angewandt worden sein".40 Die Romanze hat nach Schlegel ihre Dominante und ihr bestimmtes Kolorit. Sie erscheint ihm romantisch und dem Ritterepos vergleichbar, nur dass sie sich auf einzelne Tatsachen beschränkt.

Die spanischen Romanzen sind im schlichtesten, einfachsten Volkstone, oft haben sie etwas von der Novelle, etwas Seltsames, Kontrastierendes, das den Vorfallen Reiz und Anlockung gibt. Die Romanze muss bei jeder Nation nationa $\operatorname{sein} 4$

Schlegel stimmt nicht mit Herder überein, nach dem die spanischen Romanzen die ältesten seien. Ihm scheinen sie nicht hinter das 15. Jahthundert zurückzugehen. Immerhin sind die Romanzen der Ort der Poesie "als sie sich mehr und mehr gegen Ende des sogenannten Mittelalters aus den höheren Ständen verlor, unter dem Volke einen Zufluchtsort fand" ". 42

An zentralerer Stelle als die Romanze steht bei Schlegel das spanische Theater, das sich ganz ohne Einflüsse aus fremden Ländern wie Italien oder Frankreich entwrickelt habe. ${ }^{43}$ Ganz im Gegenteil, bei italienischen, französischen und englischen Dramatikern sei es mehr oder weniger Sitte gewesen, aus spanischen Quellen zu schöpfen, meistens ohne sie explizit anzugeben, wenngleich sie deutlich zu erkennen sind:

Sinnreiche Kühnheit mit leichter Klatheit in der Intrige vereinigt, ist den spanischen Dramatikern so ausschließend eigen, dass ich mich berechtigt halte, wo ich sie in einem Lustspiele finde, einen spanischen Ursprung zu vermuten, wen

38 Lohner VI, (Aлm. 1), S. 112.

39. Der Kontrast von Schetz und Enst, vorausgesert daß sie im Grade und der Art ein Verhälnis zueinander haben"; Ebd., S. 113.

KAV II/1, (Anm. 2), S. 125.

41 August Wilhelm Schlegel, „Vorlesungen über Ästhetik [1798-1803]“, in: KAV I, hier S. August

42 KAV II/1, (Anm. 2), S. 124

43 Vgl. Lohner VI, (Anm. 1), S. 107 auch der Verfasser selbst es nicht wusste, sondern ein Plagiat an einer nähern Stelle verübte. 44

Das englische Theater Shakespeares allerdings ist dem spanischen gleichrangig, wie Schlegel mehrfach hervorhebt. Manchmal sind für ihn Shakespeare und Calderón die einzig großen Dichter, ${ }^{45}$ manchmal aber gibt er Shakespeare den Vorzug. ${ }^{46}$ Das englische und spanische Theater verbinde nicht nur die Vernachlässigung der Einheiten und die Vermischung komischer und tragischer Bestandteile, die, wie bereits erwähnt, Lessing in der Hamburgischen Dramaturgie hervorgehoben hatte, ${ }^{47}$ sondern ein gemeinsamer innerster Gehalt der sich auch als der Geist der romanischen Poesie" bezeichnen lasse. ${ }^{48}$ Umso erstaunlicher sei es, dass bei der Theaterentwicklung zweier Völker, „die in physischer, moralischer, politischer und religiöser Hinsicht so weit voneinander abstehen, wie die Engländer und die Spanier", ein gleichartiges Prinzip zugrunde liege,49 das weder Tragödien, noch Komödien, sondern romantische Schauspiele hervorbringe.

Cervantes, der sich als Theaterautor noch an den aristotelischen Regeln orientiert, geht nach Schlegel am Zeitgeschmack vorbei, wenngleich auch die Meinung vertreten werden könne, dass seine Schauspiele „Parodien und Satiren auf den verderbten Zeitgeschmack ${ }^{\star 50}$ seien. Gemessen an Lope de Vega oder Calderón erscheint Cervantes jedoch weniger talentiert. Lope de Vega dagegen wird von Schlegel als Wunder der Natur gelobt, jedoch kritisiert, da es seinen Werken „an Tiefe und an jenen feineren Bezichungen, welche eigentlich die Mysterien der Kunst ausmachen", fehle. 51

Größter Theaterautor Spaniens ist für Schlegel Calderón de la Barca. „In ihm hat das romantische Schauspiel der Spanier den Gipfel der Vollendung erreicht" ${ }^{.52}$ Calderóns Biographie präge das 17. Jahrhundert: Er war sechzehn Jahre alt, als Cervantes, und fünfunddreißig, als Lope starb, den er fast um ein halbes Jahrhundert überlebte. Zentrale Themen in Calderóns Comedias de capa y espada sind Ehre, Liebe und Eifersucht. Insgesamt lassen sich Calderóns Stücke in folgende Untergattungen gliedern: „Darstellungen heiliger Geschichten aus der Schrift und der Legen-

4 Lohner VI (Anm. 1) S. 251

45 Ebd., S. 109

46 KAVI, (Anm. 41) S. 114

47 Vgl. Paulini, Vergleichende Literaturwissenschaff, (Anm. 3), S. 254.

48 Lohner VI, (Anm. 1) S. 111.

49 Ebd., S. 110

50 Ebd., S. 254

51 Ebd., S. 257; vgl. auch Paulini, Vergleichende Literaturnissensschaft, (Anm. 3), S. 258.

52 Lohner VI, (Anm. 1) S. 260 . 
de; historische; mythologische oder aus anderen erdichteten Stoffen gebildete; endlich Schilderungen des geselligen Lebens in modernen Sitten". 53 Ein besonderer Reiz des.Theaters bestand nach Schlegel darin, die schon halbverlorene welterobernde Größe der spanischen Nation vorzuführen. ${ }^{54}$ Calderón hat nach Schlegel das Theater so nachhaltig geprägt, dass noch lange nach ihm „nur Nachklang des Vorhergehenden, [...] nichts Neues, noch wahrhaft Eigentümliches zum Vorschein gekommen" 55 sei. Als in anderen Ländern Europas schon die aufklärerischen Tendenzen des 18. Jahrhunderts herrschten, blühte nach Schlegel in Spanien noch das Werk Calderóns: „Er ist folglich als der letzte Gipfel der romantischen Poesie zu betrachten". .56

Eine gewisse Verwandtschaft mit dem Drama hat nach Schlegel die Novelle. Wenn er die Gattung definiert, bezieht er sich paradigmatisch auf die Novellen von Cervantes. Die Novelle erzähle merkwürdige Begebenheiten. Dazu gehöten teils seltsame, bald günstige, bald ungünstige Wechselfälle des Glücks, teils schlaue Streiche, die der Befriedigung der Leidenschaft dienen. Erstere bezeichnet Schlegel als tragisch, letztere als komisch, da dabei "das sittlich zu Missbilligende durch den Aufwand von Scharfsinn bei der Erfindung geheimer Mittel zu einem Zweck, durch die Gegenwart des Geistes beim Ausführen, und den Witz womit die Gesinnung geäußert wird“.57 Als Dramatisierung von Novellen sei auch Shakespeares Theaterschaffen zu kennzeichnen. ${ }^{58}$ Gleichgïltig, ob die Novelle tragisch oder komisch ist, sie hat realistisch zu sein, Ort, Zeit und Personennamen anzugeben und den Menschen „mit allen Schwächen, Leidenschaften und selbstischen Trieben, welche der ungeläuterten Natur anhängen " ${ }^{59}$ darzustellen.

Ein besonders wichtiger Gipfelpunkt der spanischen Literatur ist für Schlegel Cervantes. So sehr war er Dichter, „daß selbst seine Vorreden

53 Ebd., S. 258.

54 Vgl. Reavis, Scblegels Auffassung der Tragödie, (Anm. 23), S. 135; vgl' auch Paulini, Vergleichende Literaturnissenschaft, (Anm. 3), S. $257 \mathrm{f}$

5 Lohner VI, (Anm. 1), S. 267

56 Ebd., S. 266

57 August Wilhelm Schlegel, „Geschichte der tomantischen Litetatur", in: Lohner IV, hier S. 219.

58 Ebd., S. $215 f$

59 „Die Novelle kann von ersten Begebenheiten mit tragischer Katastrophe bis zur bloßen Posse alle Töne durchlaufen, aber immer soll sie in der wirklichen Welt zu Hause sein, deswegen liebt sie auch die ganz bestimmten Angaben von Ort Zeit und Namen der Personen. Daher muss sie den Menschen in der Regel nach seinem Naturstande nehmen, d.h. tmit allen Schwächen, Leidenschaften und selbstischen Trieben, welche der ungeläuterten Natur anhängen. Sie soll den Weltauf schildern, wie er ist; sie darf also die Motive im allgemeinen nicht über Gebühr veredeln". Ebd., S. 218. und Zueignungen [...] wahre dichterische Kompositionen sind ".60 Seine Dichtung ist „ein vollendetes Meisterwerk der höheren romantischen Kunst".61 Cervantes' Don Ouijote ist nach Schlegel voller romantischer Darstellungen und hat Romantisches im Gehalt. Schnell und erfolgreich wurde das Werk in Deutschland und England verbreitet, wobei zunächst die Deutung als komisches Werk vorgeherrscht habe. Der verbreiteten Mode der Ritterbücher begegne Cervantes mit Spott. „Indem der Dichter die abgeschmackte und kolossale Romanwelt der Ritterbücher zerstört erschafft er auf dem Boden seines Zeitalters und einheimischer Sitten eine neue tomantische Sphäre". ${ }^{62}$

Zusammenfassend hält Schlegel fest, dass es im Roman darauf ankomme, dass die Episoden in ihrem Wechsel harmonisch und phantasievoll seien.

Wenn je ein Roman dies auf das vollkommenste geleistet hat, so ist es Don Quixote. So erkennt man überall den besonnenen Künstler in der weisen Anordnung und Verteilung. Gleich bei Eintritt lässt er die überspannten Ideen des Ritters, um ihm gat keinen Schlupfwinkel zur Rettung übrigzulassen, gegen die gemeinste Wirklichkeit anstoßen. ${ }^{63}$

Abschließend soll nun in einem ausführlicheren Exkurs August Wilhelm Schlegels Deutung des Don Quijote kontextualisiert werden. Zunächst sei sein Bruder Friedrich Schlegel erwähnt. Man ist gewohnt, ihn als Essayisten und Literaturtheoretiker zu sehen. Bedenkt man aber, dass er auch in die philosophischen Diskussionen seiner Zeit eingegriffen und die idealistische Philosophie besonders gelobt hat, dann müssen auch seine literaturtheoretischen Ausführungen vor philosophischem Hintergxund gesehen werden. Es darf auch nicht vergessen werden, dass von ihm ein Buch zur Transzendentalphilosophie erschienen ist und er sich auch mit der Lehre Fichtes auseinandergesetzt hat. ${ }^{64}$ Als Fichtes Verdienst erkennt er 1808, dass er

die in der Denkart des Zeitalters begründete empirische Beschränktheit, welche Kant noch hatte stehen lassen, die Kantianer aber noch weiter ausgedehnt und noch mehr befestigt hatten, bis auf die Wurzel zerstörte; und daß er zugleich zeigte, welche mächtige Wirkung ein freierer und kühnerer Gebrauch der Idee zeigte, welche mächtige Wirkung ein freiere
des Unendlichen hervorbringen könne.65

60 Staiger, S. 299.

61 Ebd., S. 294.

62 Ebd., S. 295

63 Ebd., S. 296.

64 Vgl. Friedrich Schlegel, Transcendentalpbilosopbie, Hamburg 1991; Friedrich Schlegel, Kritische Scbriften und Fragmente, Ernst Behler/Hans Eichner (Hrsg.), Bd. 3, Paderborn, Münche 1988, S. 109-125.

65 Ebd., S. 109. 
Den Grundgedanken der Fichteschen Lehre formuliert Friedrich Schlegel so:

[D]ie Natur als tote Sinnenwelt und bloßet Niederschlag der Reflexion sei das eigentliche Nichtsein, ein durchaus nichtiges und ganz und gar ungöttliches Scheinwesen; bloß Hemmung und Schranke des sich ins Unendliche fortentwickelnden Geistes. 66

Zur Bedeutung der idealistischen Philosophie schreibt F. Schlegel, dass in einer Zeit, in der die Sitten entartet, die Gesetze verdorben und alles verwirrt und verfälscht sei, ,durch Philosophie allein, die Wohlfahtt der Menschen wieder hergestellt und aufrecht erhalten werden" ${ }^{66}{ }^{67}$ Gerade die neue Philosophie des Idealismus zeige das "Äußerste, was der Mensch bloß durch sich selbst vermag, durch die Kraft und Kunst des freien Denkens allein, und durch den festen Mut und Willen dazu, in steter Befolgung der einmal erkannten Grundsätze".68 Während im ersten Beleg die reale Wirklichkeit als moralisch negativ kritisiert und ihr die positiv bewertete geistige Wirklichkeit des philosophischen Idealismus gegenübergestellt wird, ist es im zweiten Beleg eben das im Sinne des Idealismus denkende Subjekt, dessen schöpferische Kraft hervorgehoben wird. Nach Friedrich Schlegel ist daher der Idealismus der

Mittelpunkt und die Grundlage der deutschen Literatur; ohne ihn ist eine das Ganze der Natur umfassende Physik nicht möglich, und die höhere Poesie als ein andrer Ausdruck derselben transzendentalen Ansicht der Dinge ist nur durch die Form von ihm verschieden. ${ }^{69}$

Es gibt also eine gewisse Affinität zwischen der Deutung des Don Quijote als Verkörperung des Strebens nach Unendlichkeit und der idealistischen Philosophie, auf die kurz eingegangen werden soll. Deren Ausgangspunkt ist relativ einfach: So wie der absolute Geist die Welt geschaffen hat, so erschafft ganz analog das individuelle Subjekt seine Realität aus sich heraus und mit seiner geistigen Gestaltungskraft. Aktivität und Gestaltung gehen also vom Subjekt aus, sodass die Realisierung des Objekts dem Subjekt zu verdanken ist. Schelling formuliert das so: „Gott ist das, was keinen anderen Begriff voraussetzt, wie der Raum in der Geometrie. Die Welt hingegen kann nur als Folge von ihm begriffen werden“".70 Das

66 Ebd., S. 113. Allerdings entwickelte sich Schlegels Philosophie immer mehr zur Gegenbewegung gegen die Hochschätzung der Vernunit in der Aufklarung und in der darauf folgenden Philosophie Hegels. Die Aktuierung des subjektiven Bewusstseins ist für Schlegel daher eine unbewusste Betätigung des Bewusstseins. VgL Michael Elsässers „Einleitung in: Schlegel, Transcendentalpbilosopbie, (Anm. 64), S. XVE.

67 Ebd., S. 77

68 Ebd.

69 Friedrich Schlegel, „Literatur", in: KFSA III, S. 3-16, hier S. 5.

70 Friedrich Wilhelm Joseph Schelling, Einleitung in die Philosopbie, Stuttgart 1989, S. 68 göttliche Wollen wird zum Anfang der Natur, schafft also die Natur. ${ }^{71}$ So ist Gott jene Ursache im Weltprozess, ,die dem Idealen über das Reale Übergewicht verleiht".72 Was für Gott gilt, ist analog auch für die Geschöpfe richtig: „Die endlichen Wesen hingegen haben nur die Freiheit, sich ewig selbst zu setzen. ${ }^{473}$ Dies bedeutet, dass die Realität explizit darstellt, was in der Idee implizit bereits vorgegeben ist. Die Natur entsteht dadurch, dass die in der Idee enthaltenen Momente auseinander fallen und nebeneinander bestehen. „Die Natur ist nach dieser Lehre nichts, als die auseinandergefallene Idee ${ }^{\text {" } 74 ~-~, ~, d e n n ~ j e d e s ~ W e s e n ~ v e r w i r k-~}$ licht sich nur dadurch, daß es das implizierte Sein als expliciter setzt ${ }^{\text {“ }} .{ }^{75}$ Die innere Welt hat also für Schelling Priorität vor der äußeren:

D]ie wahre Tatsache ist aber immer etwas Innerliches; die wahre Tatsache einer Schlacht z.B. liegt in dem Geiste des Feldherrn, nicht in den Angriffen odet $\mathrm{Ka}$ nonenschüssen; die wahre Tatsache eines Buches kennt nur derjenige, welcher es versteht. ${ }^{76}$

Zur Veranschaulichung führt Schelling Geometrie und Licht an. In beiden Beispielen geht etwas von einem Punkt aus und legt sich auf die Objekte der Realität, seien es die geometrischen Modelle, seien es z.B. die Lichtstrahlen der Sonne. Das Licht könne nicht Materie sein, da es durchsichtige Körper durchbohre. „Das Licht ist doch wohl in der ausgedehnten Welt ein Analogon des Geistes, ja das Licht ist gar nichts anderes, als der denkende Geist, nur auf seiner tiefsten Stufe “ 77

Charakteristisch für Hegels philosophischen Standpunkt ist nun, dass er nicht etwa das Subjekt durch die Ordnung der Dinge korrigiert wissen will, sondern am Subjekt festhält auf Kosten der Dinge. Hegel bezeichnet Don Quijote als edlen Charakter, hineinversetzt in den festen, bestimmten Zustand einer ihren äußeren Verhältnissen nach genau geschilderten Wirklichkeit. Daraus ergibt sich der

Widerspruch einer verständigen, durch sich selbst geordneten Welt und eines isolierten Gemütes, das sich diese Ordnung und Festigkeit erst durch sich und das will. [..] Don Quijote ist ein in der Verrücktheit seiner selbst und seiner Sache vollkommen si-

71 Vgl. ebd., S. 86

72 Ebd., S. 97

73 Ebd., S. 106

74 Ebd., S. 65.

75 Ebd, S. 53 .

76 Ebd., S. $37 f$

77 Ebd., S. 51; Bei der Geometrie beruft sich Schelling auf ein Gleichnis von Platon, wonach "die Geometrie von einem wahthaft Seienden nur träume, aber es nicht erreiche“. Ebd., S. 18. 
cheres Gemüt, oder vielmehr ist nur dies die Verrücktheit, daß er seiner und seiner Sache so sicher ist und bleibt. 78

Die Sympathie Hegels gilt also dem isolierten ritterlichen Gemüt des Don Quijote, der durch das Rittertum eine eigene Ordnung schaffen und die vorhandene, durch sich selbst geordnete Welt umstürzen will. Don Quijote ist also ein Paradigma für das Subjekt, das sich das Objekt in seinem Geist erschafft

Bei der Wertschätzung des Rittertums, seiner Abenteuer und Kollisionen konnte Hegel auf Friedrich Schlegel zurückgreifen, für den das Rittertum ebenso spanisch wie romantisch ist

$\mathrm{Da}$ die spanische Dichtkunst überhaupt ohne allen fremdartigen Einfluß und durchaus rein tomantisch geblieben ist, da die christliche Ritterpoesie des Mittelalters dieser Nation am längsten bis in die Zeiten der neuern Bildung fortgedauert, und die kunstreichste Form erlangt hat, so ist hier wohl der rechte Ort, das Wesen des Romantischen überhaupt zu bestimmen. ${ }^{79}$

Für besonders wertvoll in der älteren spanischen Literatur hält Friedrich Schlegel die Ritterbücher, wie den Amadís, deren romantische Ausgestaltung und idyllischen Charakter er bei den Spaniern hervorhebt. Spuren dieser Ritterbücher seien noch in Cervantes' Don Ouijote erkennbar. ${ }^{80}$

Wenn Schelling, wie sich gezeigt hat, Cervantes als modernen Autor auf dieselbe Stufe stellt wie Homer als antiken Autor, dann ist dies nicht zuletzt darin begründet, dass beide Mythen geschaffen haben. Mythen sind als Objektivietungen des Geistes in der Philosophie des deutschen Idealismus von besonderer Bedeutung. Aus der Tatsache, dass die antike Literatur immer wieder aus ihren Mythen schöpfen konnte, leitet Friedrich Schlegel den Nachteil der modernen Dichtung ab, der darin bestehe, dass sie derartige Mythen nicht habe. Prägnant formuliert er: "Wir haben keine Mythologie. Aber, setze ich hinzu, wit sind nahe daran, eine zu erhalten, oder vielmehr, es wird Zeit, daß wir ernsthaft dazu mitwirken sollen, eine hervorzubringen". ${ }^{81}$ Diese neue Mythologie hat sich nun auf der Basis des philosophischen Idealismus aufzubauen. Neuere Mythen sieht Schelling

Georg Wilhelm Friedrich Hegel, Ästhetik, Bd. 1, Frankfurt/Main 1955, S. 566

Schlegel, Kritische Scbriften und Fragmente, (Anm. 64), Bd. 4, S. 160

80 Friedrich Schlegel, „Beiträge zur Geschichte der modernen Poesie und Nachricht von provenzalischen Manusktipten", in: KFSA III, S. 17-37, hier S. 21. Ebenso wie im Idealismus der subjektive Geist das Objektive gestaltet, sei es in der Literatur die Poesie, die die prosaische Realität ungestaltet, indem sie sich ihr überstülpt. Schlegel konktetisiert das in seiner Besprechung der Don Qunijote-Ubersetzungen. Während es den bisherigen Ubersetzungen an Poesie gefehlt habe, ist es Tieck als vertrauter Freund "der alten tomantischen Poesie", „der diesen Mangel ersetzen und den Eindruck und Geist des Ganzen im Deutschen wiedergeben und nachbilden will“" Friedrich Schlegel, „Über Tiecks Don Quixote“, in: KFSA II, S. 281-283, hier S. 281.

81 Friedrich Schlegel, „Rede über die Mythologie“, in: KFSA II, S. 311-322, hier S. 312 im Roman: „Der Roman soll ein Spiegel der Welt, des Zeitalters wenigstens, seyn, und so zur partiellen Mythologie werden".82 Und so nennt Schelling Don Quijote, um zu zeigen,

was der Begriff von einer durch das Genie eines Einzelnen erschaffenen Mythologie sagen will. Don Quijote und Sancho Pansa sind mythologische Personen über den ganzen gebildeten Erdkreis, sowie die Geschichte von den Windmühlen u.s.w. wahre Mythen sind, mythologische Sagen. 83

Thema des Don Quijote und damit Philosophem ist das Ideale im Kampf mit dem Realen. Der kleine Exkurs zur Don Quijote-Rezeption sollte zeigen, inwiefern dieser Roman nicht nur als Identifikationsmuster für romantische Dichtung, sondern auch für die Philosophie des Idealismus dient.

Abschließend sei ein Sonett von August Wilhelm Schlegel zitiert, das sich mit dem Philosophem Don Ouijote, d.h. dem Kampf des Idealen mit dem Realen, in humorvoll satirischer Weise auseinandersetzt:

Auf seinem Pegasus, dem magern Rappen

Reit' in die Ritterpoesie Quixote,

Und hält anmuthiglich, in Glück und Nothe

Gespräche mit der Prosa seines Knappen.

Erst, wie sie blind nach Abenteuem tappen,

Trifft sie der Weltlauf mit gar harter Pfote;

Dann kommt der Schetz als huldigender Bote,

Und schüttelt schelmisch ihre Schellenkappen.

Und Liebe webt drein rührende Geschichten;

Verstand der Menschen Sitten, Tracht, Geberden

Es gaukelt Phantasie in farb'ger Glorie.

Ich schwör'es, und Urgande selbst soll richten:

Was auch hinfüro mag exsonnen werden

Dieß bleibt die unvergleichlichste Historie

82 Schellings Werke, Bd. 3, Manfred Schröter (Hrsg.), München 1959, S. 327

83 Ebd., S. 330.

84 Böcking I, S. 342 